
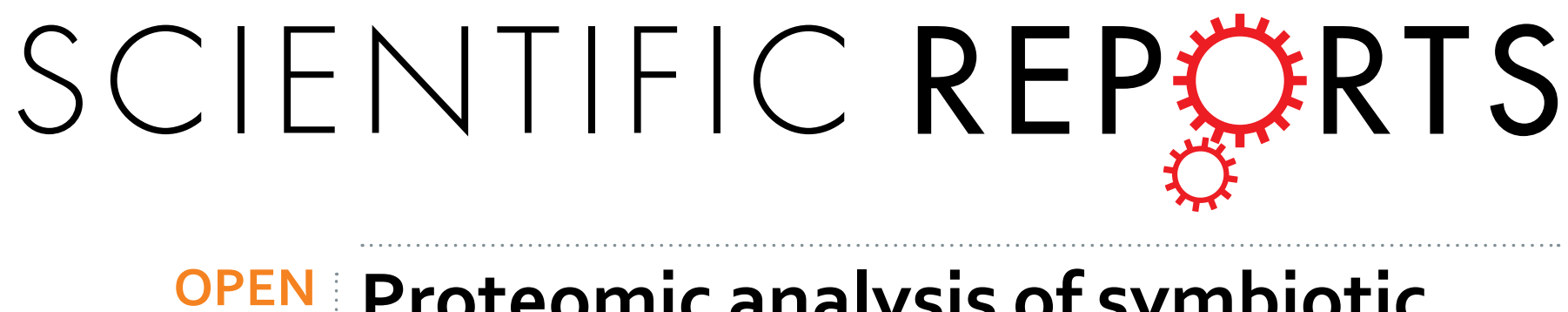

\title{
Proteomic analysis of symbiotic proteins of Glomus mosseae and Amorpha fruticosa
}

Received: 30 June 2015

Accepted: 10 November 2015

Published: 10 December 2015

\author{
Fuqiang Song, Dandan Qi, Xuan Liu, Xiangshi Kong, Yang Gao, Zixin Zhou \& Qi Wu
}

Arbuscular mycorrhiza fungi (AMF) can colonize the roots of Amorpha fruticosa, a perennial leguminous woody shrub, and form arbuscular mycorrhiza (AM). AMF have significant promoting effects on $A$. fruticosa growth as the intensity of fungal colonization increases. Taking AMF-A. fruticosa symbionts as the experimental material, gel-free isobaric tags for relative and absolute quantification (iTRAQ) coupled with two-dimensional liquid chromatography-tandem mass spectrometry (LC-MS/MS) were used to investigate the expression of $A$. fruticosa mycorrhizal proteins at the maturation stage. A total of 3,473 proteins were identified, of which 77 showed dramatic changes in their root expression levels; 33 increased, and 44 decreased. We also found nine AMF proteins that were expressed with AMF treatment. The 77 proteins were classified according to function. Plant proteins were assigned into 11 categories: metabolism-related (32\%), protein folding and degradation-related (22\%), energyrelated (10\%), protein synthesis-related (8\%), stress and defense-related (24\%), transcription-related $(6 \%)$, membrane and transport-related (4\%), cellular structure-related $(2.5 \%)$, signaling transductionrelated (11\%) and unknown proteins (5\%). The results of the study provide a foundation for further investigation of the metabolic characteristics and molecular mechanisms of AM.

Arbuscular mycorrhiza (AM), representing the most widely distributed mutualistic root symbiosis in nature, are the result of long-term evolution between plant and soil fungi. AM fungi (AMF) are obligate symbionts and are recalcitrant to pure culture on synthetic media; they grow only in living plants. Their hereditary variability and heterogeneous characteristics are essential for colonizing a large number of potential host plants. However, different host plants may simultaneously induce the expression of different symbiosis-related genes. AMF are some of the most widespread microorganisms, and they can form symbionts with more than two-thirds of the vascular plants in natural or artificial ecosystems. These plants include important agricultural species, such as wheat, rice, and the model plant Populus trichocarpa $a^{1,2}$. The foundation of mycorrhizal symbiosis is the ability of AMF, using their multicore hyphae, to provide nutrients (especially phosphorous) to host plants that have long-distance illiquidity $^{3}$. New physiological and molecular evidence has shown that, for phosphorus, the mycorrhizal pathway (MP) is operational regardless of plant growth responses. Meanwhile, the contribution of the direct pathway (DP) is decreased, which results in a greater dependence of host plants on the nutrients that AMF provide ${ }^{4}$. AMF can utilize only simple carbon and nitrogen sources from their hosts to complete their life cycles. This may be due to the loss of some enzyme-encoding genes and to macromolecular synthesis defects that have arisen during the long-term evolution of symbiosis with plants ${ }^{5,6}$.

AM play a significant role in promoting the growth of host plants, and researchers have increased their efforts to study the interactions between AMF and host plants. Recorbet and colleagues have compared the root proteome responses of Medicago truncatula upon colonization with two AM fungi, i.e., Glomus mosseae (GM) and G. intraradices, using two-dimensional electrophoresis (2-DE) ${ }^{7}$. They found 42 symbiosis proteins; of these, 32 could be confidently identified and retrieved following MS/MS and matching with a database encompassing 21 fungal proteins. To test the mechanisms by which shoots of Cd-treated mycorrhizal plants avoid metal toxicity, Aloui has performed a 2-DE/MALDI-TOF-based comparative proteomic analysis of the $M$. truncatula shoot responses upon mycorrhization and $\mathrm{Cd}$ exposure ${ }^{8}$. finding that $\mathrm{Cd}$ triggers an opposite response than mycorrhization, which is coupled with an increase in molecular chaperones in the shoots of mycorrhizal plants relative to those that are metal-free. Wang has studied the dynamic changes in maize leaf protein expression profiles under AMF colonization ${ }^{9}$. In that study, the differentially expressed proteins in maize leaves were separated by 2-DE,

Heilongjiang University, Harbin, Heilongjiang, China. Correspondence and requests for materials should be addressed to F.S. (email: 0431sfq@163.com) 


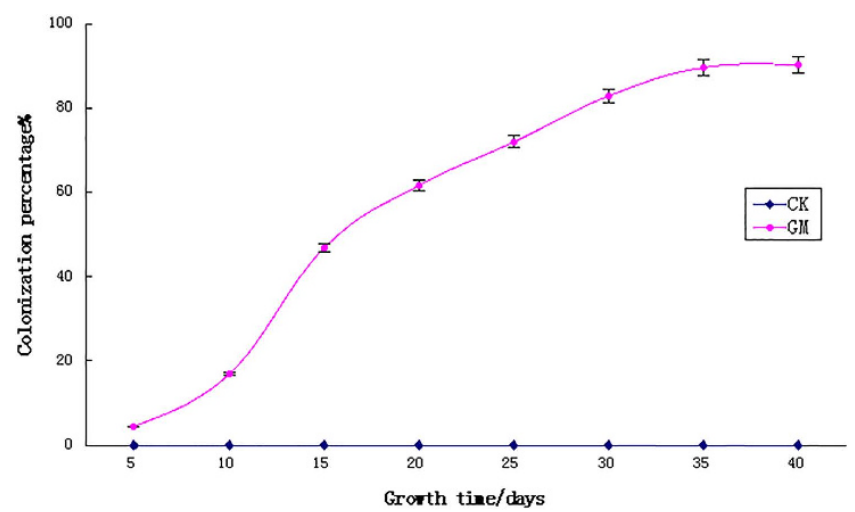

Figure 1. The colonization percentage of Amorpha fruticosa roots by AMF changes with time.

and the results reveal 21 differentially expressed gel spots in maize leaves. Among them, 8 proteins were successfully identified. With the development of molecular biology techniques, quantitative analysis of the differences in protein expression profiles during the colonization process of pathogenic or symbiotic microorganisms has become possible; these techniques have played a critical role in analyzing pathogenic mechanisms. Isobaric tags for relative and absolute quantification (iTRAQ) represents one of the new and powerful techniques for simultaneous analysis of multiple samples and provide relative quantification of hundreds of proteins. iTRAQ reagents produce high-quality, reproducible results from complex samples, and iTRAQ has thus become widely used. However, iTRAQ-based studies on the symbiotic mechanisms of AMF and host plants have rarely been reported. Our group has studied the symbiotic relationship between plants and fungi at the mRNA level, Zhang xingxing identified 30 symbiosis-related genes expressed in Amorpha fruticosa roots colonized by GM at different stages by using mRNA differential-display PCR (DDRT-PCR). The expressed genes were confirmed by reverse Northern blotting. Eleven fragments were sequenced and putatively identified by homologous alignment, and these genes were found to relate to defense and signal transduction ${ }^{10}$. Kong xiangshi also has found 47 symbiosis-related unigenes during AMF treatment by using suppression subtractive hybridization (SSH) and subsequent Gene Ontology (GO) database, BLAST annotation and literature searches to categorize each of the identified genes. Among the expressed genes, those related to plant metabolism and stress and defense show important roles during the symbiotic process of AMF-A. fruticosa ${ }^{11}$. Based on our previous work, we have continued to use A. fruticosa, a perennial leguminous woody shrub plant, as a host. Using AMF-A. fruticosa symbionts as the experimental materials, we used iTRAQ combined with 2-D LC-MS/MS to investigate the expression of $A$. fruticosa mycorrhizal proteins at the maturation stage. The results of the study provide a theoretical basis for the further analysis of the metabolic characteristics and molecular mechanisms of symbiosis between AMF and A. fruticosa.

\section{Results}

AMF Colonization. The colonization percentage of A. fruticosa roots is shown in Fig. 1. At day 5, the roots were relatively small, and only a few hyphae were detected. Most of the spores were not in contact with the host roots and were mainly in their vegetative growth stage ${ }^{12,13}$. The infection rate began to increase at day 10 , and the growth status of GM-inoculated seedlings was significantly better than that of non-inoculated plants. The colonization percentage increased rapidly at day 15 . At that time, there was a large quantity of mycelium-infected roots that increased over time. The colonization percentage reached its peak at the vigorous phase (i.e., 30 days), and a large number of vesicles and arbuscules were observed within the roots. At 40 days, we observed that the hyphae had extended from the plant root surface and had infected neighboring roots. No colonization by AM was observed in the non-inoculated plants, because the mixed soil used for culture had been autoclaved thoroughly.

Identification of Symbiosis-Related Proteins using iTRAQ LC-MS/MS. The roots of $A$. fruticosa changed their protein levels when colonized by GM, and mutualistic symbionts formed. Using an iTRAQ approach, 86 differentially expressed symbiosis-related proteins were successfully identified. Among them, 77 were plant proteins, with 33 proteins showing increases and 44 showing decreases (Table 1), and 9 were fungal proteins (Table 2). More detailed information in supplementary information. AMF proteins play an import role in symbiotic systems, but they show high expression in only the AMF themselves. The low overall concentration of AMF proteins, and the limitations of the technology, resulted in few AMF proteins being detected ${ }^{14}$.

Classification of Symbiosis-Related Proteins. The GO database, BLAST annotations and information reported in the literature were used to categorize each of the identified proteins ${ }^{11}$. The 77 differentially expressed proteins in A. fruticosa were categorized into different functional classes and assigned to 11 categories. The functional categories are shown in Fig. 2; they include metabolism-related (32\%), protein folding and degradation-related (22\%), energy-related (10\%), protein synthesis-related (8\%), stress and defense-related $(24 \%)$, transcription-related (6\%), membrane and transport-related (4\%), cellular structure-related (2.5\%), signaling transduction-related (11\%) and unknown (5\%). Among these classes, proteins related to plant metabolism, protein folding and degradation, and energy (totaling $64 \%$ of the identified proteins) play important roles during the symbiotic process of AMF-A. fruticosa. 


\begin{tabular}{|c|c|c|c|c|}
\hline Accession & Protein name & Plant species & $\begin{array}{l}\text { Unused } \\
\text { score }\end{array}$ & FM/control \\
\hline \multicolumn{5}{|l|}{ Metabolism } \\
\hline 356523620 & 3-oxoacyl-[acyl-carrier-protein] synthase I & Glycine max & 6 & 6.88 \\
\hline 502118474 & chorismate synthase, chloroplastic-like isoform X1 & Cicer arietinum & 2.2 & 1.96 \\
\hline 17026394 & UDP-glucose pyrophosphorylase & Amorpha fruticosa & 52 & 0.43 \\
\hline 502122125 & pyruvate kinase, cytosolic isozyme & Cicer arietinum & 26 & 0.51 \\
\hline 502134148 & 5-methyltetrahydropteroyltriglutamate--homocysteine methyltransferase & Cicer arietinum & 24 & 0.52 \\
\hline 356505594 & sucrose synthase 2 & Glycine max & 22 & 0.43 \\
\hline 356551144 & alpha-1,4 glucan phosphorylase $\mathrm{L}$ isozyme & Glycine max & 22 & 0.33 \\
\hline 357453895 & 4-hydroxy-3-methylbut-2-en-1-yl diphosphate synthase & Medicago truncatula & 20 & 0.51 \\
\hline 356557483 & carbamoyl-phosphate synthase large chain & Glycine max & 20 & 0.53 \\
\hline 356576733 & zeta-carotene desaturase, chloroplastic/chromoplastic & Glycine max & 20 & 0.51 \\
\hline 356542858 & beta-amylase & Glycine max & 19 & 0.27 \\
\hline 502081957 & UDP-glucuronic acid decarboxylase 6-like isoform X3 & Cicer arietinum & 14 & 0.67 \\
\hline 17063848 & 4-coumarate:CoA ligase & Amorpha fruticosa & 14 & 0.47 \\
\hline 356566195 & alpha-glucan phosphorylase, Hisozyme & Glycine max & 13 & 0.32 \\
\hline 356552274 & glucose-1-phosphate adenylyltransferase small subunit & Glycine max & 12 & 0.49 \\
\hline 502151454 & probable sucrose-phosphate synthase & Cicer arietinum & 12 & 0.54 \\
\hline 355481047 & Neutral invertase & Medicago truncatula & 11 & 0.29 \\
\hline 356536154 & methylthioribose kinase & Glycine max & 8.6 & 0.46 \\
\hline 357445053 & Leukotriene-A4 hydrolase & Medicago truncatula & 7.6 & 0.17 \\
\hline 84514155 & p-coumaroyl-shikimate 3'-hydroxylase & Trifolium pratense & 6.7 & 0.19 \\
\hline 502131944 & carotenoid 9,10(9',10')-cleavage dioxygenase 1-like isoform X1 & Cicer arietinum & 6.2 & 0.08 \\
\hline 68264915 & beta-conglycinin alpha subunit & Glycine max & 5 & 0.20 \\
\hline 356509826 & thromboxane-A synthase & Glycine max & 5.2 & 0.78 \\
\hline 356549592 & caffeic acid 3-O-methyltransferase & Glycine max & 2.8 & 3.91 \\
\hline 502134043 & neutral ceramidase & Cicer arietinum & 6.3 & 12.65 \\
\hline \multicolumn{5}{|l|}{ Signaling } \\
\hline 357446813 & Somatic embryogenesis receptor -like kinase & Medicago truncatula & 12 & 2.21 \\
\hline 502110137 & rho GDP-dissociation inhibitor 1 & Cicer arietinum & 2.4 & 2.86 \\
\hline \multicolumn{5}{|c|}{ Stress and defense } \\
\hline 356523918 & agglutinin-2 & Glycine max & 5.8 & 11.47 \\
\hline 356527464 & transaldolase & Glycine max & 17 & 0.52 \\
\hline 310704426 & stearoyl-acyl carrier protein desaturase & Phaseolus lunatus & 5.6 & 0.54 \\
\hline 356518971 & pantothenate kinase 2 & Glycine max & 5.9 & 0.61 \\
\hline 502180482 & abscisic acid $8^{\prime}$-hydroxylase 3 & Cicer arietinum & 2.4 & 13.80 \\
\hline 357438243 & Germin-like protein subfamily 1 member & Medicago truncatula & 2.4 & 16.44 \\
\hline \multicolumn{5}{|c|}{ Membrance and transport } \\
\hline 356526157 & coatomer subunit beta' -2 & Glycine max & 16 & 1.58 \\
\hline 356532026 & $\mathrm{~K}(+)$ efflux antiporter 2, chloroplastic & Glycine max & 1.5 & 24.89 \\
\hline 75219328 & Protein TIC110, chloroplastic & Pisum sativum (pea) & 10 & 0.42 \\
\hline \multicolumn{5}{|l|}{ Energy } \\
\hline 356521795 & dihydrolipoyl dehydrogenase & Glycine max & 22 & 3.44 \\
\hline 356552735 & isocitrate dehydrogenase [NAD] regulatory subunit 1 & Glycine max & 2.2 & 1.67 \\
\hline 502163841 & aldehyde dehydrogenase family 2 member $\mathrm{C} 4$ & Cicer arietinum & 2.2 & 3.98 \\
\hline 48927683 & putative inorganic pyrophosphatase & Arachis hypogaea & 5.6 & 0.26 \\
\hline 372450305 & ATPase subunit 1 (mitochondrion) & Lotus japonicus & 41 & 0.30 \\
\hline 356520768 & stellacyanin & Glycine max & 2.1 & 0.43 \\
\hline 9280616 & NADH dehydrogenase subunit 9 & Lupinus angustifolius & 16 & 0.38 \\
\hline \multicolumn{5}{|c|}{ Protein folding and degradation } \\
\hline 502132065 & proteasome subunit beta type- 6 & Cicer arietinum & 21 & 2.34 \\
\hline 355518872 & Bi-ubiquitin & Medicago truncatula & 18 & 2.51 \\
\hline 356547865 & serine carboxypeptidase II-3 & Glycine max & 8.3 & 42.54 \\
\hline 356500665 & serine carboxypeptidase 24 -like isoform 2 & Glycine max & 5.7 & 3.20 \\
\hline 356540970 & serine carboxypeptidase-like 34 & Glycine $\max$ & 5 & 2.78 \\
\hline 163914235 & subtilase & Lotus japonicus & 3 & 2.72 \\
\hline 356514109 & subtilisin-like protease SDD1 & Glycine max & 2.1 & 1.31 \\
\hline
\end{tabular}




\begin{tabular}{|c|c|c|c|c|}
\hline Accession & Protein name & Plant species & $\begin{array}{l}\text { Unused } \\
\text { score }\end{array}$ & FM/control \\
\hline 502162590 & oligopeptidase A & Cicer arietinum & 17 & 0.59 \\
\hline 49257109 & protein disulfide isomerase & Glycine max & 18 & 1.28 \\
\hline 356548123 & $26 \mathrm{~S}$ proteasome regulatory subunit 4 homolog $\mathrm{A}$ & Glycine max & 15 & 0.55 \\
\hline 502142068 & $26 \mathrm{~S}$ protease regulatory subunit 8 homolog $\mathrm{A}$ & Cicer arietinum & 28 & 0.48 \\
\hline 356553349 & probable $26 \mathrm{~S}$ proteasome non-ATPase regulatory subunit 3 & Glycine max & 16 & 0.61 \\
\hline 357474441 & $26 \mathrm{~S}$ proteasome non-ATPase regulatory subunit & Medicago truncatula & 26 & 0.61 \\
\hline 502090101 & T-complex protein 1 subunit zeta & Cicer arietinum & 24 & 0.50 \\
\hline 356513012 & T-complex protein 1 subunit delta-like isoform 2 & Glycine max & 22 & 0.44 \\
\hline 357493557 & T-complex protein 1 subunit eta & Medicago truncatula & 19 & 0.58 \\
\hline 355515447 & Peptidyl-prolyl cis-trans isomerase $\mathrm{E}$ & Medicago truncatula & 6 & 0.10 \\
\hline \multicolumn{5}{|c|}{ Protein synthesis } \\
\hline 356508574 & 40 S ribosomal protein $S 9-2$ & Glycine max & 24 & 3.34 \\
\hline 356521522 & 40S ribosomal protein S16 & Lupinus polyphyllus & 10 & 1.87 \\
\hline 356571876 & Nascent polypeptide-associated complex subunit alpha & Medicago truncatula & 2.1 & 1.43 \\
\hline 2500521 & Eukaryotic initiation factor $4 \mathrm{~A}-15$ & Nicotiana tabacum & 34 & 0.62 \\
\hline 356548401 & lysyl-tRNA synthetase & Glycine max & 15 & 0.48 \\
\hline 502168167 & methionine aminopeptidase $1 \mathrm{~A}$ & Cicer arietinum & 4.1 & 0.77 \\
\hline \multicolumn{5}{|c|}{ Translation related } \\
\hline 194466266 & perchloric acid soluble translation inhibitor protein & Arachis hypogaea & 9.2 & 3.20 \\
\hline \multicolumn{5}{|c|}{ Transcription related } \\
\hline 357479669 & Histone H4 & Medicago truncatula & 14 & 6.36 \\
\hline 357485127 & Histone H3 & Medicago truncatula & 4.7 & 2.60 \\
\hline 502098976 & small nuclear ribonucleoprotein-associated protein B'-like isoform X2 & Cicer arietinum & 5.5 & 1.92 \\
\hline 356547438 & pre-mRNA-processing-splicing factor 8 & Glycine max & 31 & 0.66 \\
\hline \multicolumn{5}{|c|}{ Cellular structure } \\
\hline 502138074 & tubulin beta- 1 chain & Cicer arietinum & 8.1 & 6.32 \\
\hline 356545743 & myosin- $\mathrm{Vb}$ & Glycine max & 3.6 & 0.77 \\
\hline \multicolumn{5}{|l|}{ Unkonw } \\
\hline 291047846 & unknown & Glycine max & 22 & 0.69 \\
\hline 359807666 & unknown & Medicago truncatula & 6 & 3.37 \\
\hline 257688087 & unknown & Glycine max & 5.6 & 7.47 \\
\hline 351725945 & unknown & Glycine max & 4 & 2.03 \\
\hline
\end{tabular}

Table 1. Identified symbiosis related proteins in A. fruticosa roots colonized by G. mosseae. Note: Unused score represent this data was significant; FM/control represent the changes of more than 1.2 or less than 0.8 fold were considered as significant.

\begin{tabular}{|c|c|c|c|c|}
\hline Accession & Protein name & Fungus species & $\begin{array}{l}\text { Unused } \\
\text { score }\end{array}$ & FM/control \\
\hline \multicolumn{5}{|l|}{ Protein folding } \\
\hline 90970323 & heat shock protein 60 & Rhizophagus intraradices & 19.71 & 14.5199 \\
\hline 76780890 & binding protein & Rhizophagus intraradices & 2.21 & 16.0671 \\
\hline \multicolumn{5}{|c|}{ Cellular structure } \\
\hline 52626570 & alpha-tubulin & Glomus diaphanum & 8.82 & 18.8814 \\
\hline 219553143 & beta-tubulin & Rhizophagus clarus & 13.04 & 15.417 \\
\hline \multicolumn{5}{|l|}{ Metabolism } \\
\hline 378404947 & fumarate reductase & Rhizophagus intraradices & 11.97 & 7.7834 \\
\hline 8134607 & Phosphoglycerate kinase & Funneliformis mosseae & 3.37 & 25.881 \\
\hline 38146200 & glutamine synthetase & Funneliformis mosseae & 3.21 & 11.6025 \\
\hline \multicolumn{5}{|c|}{ Protein synthesis } \\
\hline 82792162 & $\begin{array}{l}\text { elongation factor 1-alpha, } \\
\text { partial }\end{array}$ & Scutellospora heterogama & 8.03 & 6.8289 \\
\hline \multicolumn{5}{|l|}{ Energy } \\
\hline 254212205 & $\begin{array}{l}\text { F-ATPase beta subunit, } \\
\text { partial (mitochondrion) }\end{array}$ & Glomus custos & 8.03 & 15.8362 \\
\hline
\end{tabular}

Table 2. Fungal proteins expressed in AM identified by iTRAQ approach. Note: Unused score represent this data was significant; FM/control represent the changes of more than 1.2 or less than 0.8 fold were considered as significant. 


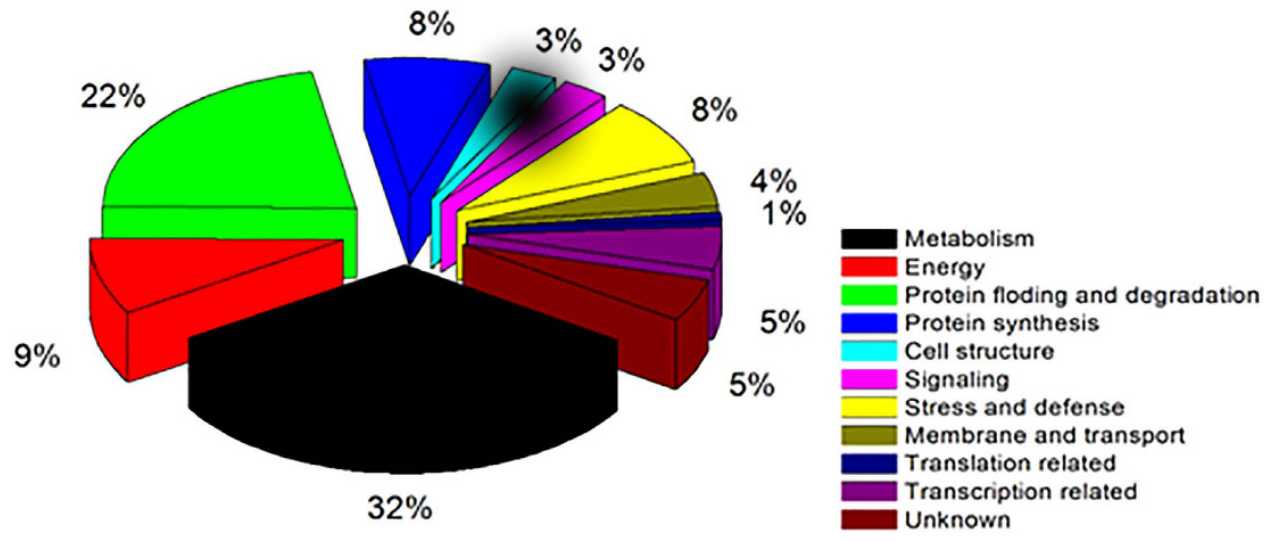

Figure 2. Functional classification of the AM symbiotic-related proteins.

\section{Discussion}

Previous studies have demonstrated that colonization is a multi-step, genetically regulated process under the control of specific loci ${ }^{15,16}$. AMF interact with host plants as cell walls, cell membranes and cellular components undergo dramatic changes ${ }^{17}$. During the colonization process, functional proteins are induced to express and regulate this process, ultimately forming stable mutualistic symbionts.

Signaling-related proteins. Mutualistic symbionts are the result of a mutual recognition and interaction process between AMF and plant signaling molecules. During the colonization process, signal transduction occurs so that the symbiotic partners recognize each other and the host plants decrease their defense responses. At the same time, AMF are prepared to colonize and to form appressoria and, subsequently, to form arbuscules, vesicles and spores. Because of their mutual nutritional relationship, a real-time dynamic signal dialogue between fungi and host plants is continually present. In this study, we found that the protein levels of Rho GDP-dissociation inhibitor 1 and somatic embryogenesis receptor-like kinase were significantly increased in the symbiotic roots.

Rho GDP-dissociation inhibitor 1, a regulator of Rho GTPase, regulates the balance of Rho GTPase bound to GTP or GDP. There are 2 conformational states of Rho GTPase: the GTP-bound 'active' state, and the GDP-bound 'inactive' state, in which GTP has been hydrolyzed to GDP ${ }^{18}$. As a member of the subfamily of small G proteins, Rho GTPase regulates a number of important signal-transduction pathways in eukaryotic cells. Rho GTPase, called Rop (Rho-related GTPase) in plants, has different isomers in animals and fungi ${ }^{19}$. Rho GTPases are widely distributed in plants, and the corresponding genes in Arabidopsis, maize, barley, rice, peas and alfalfa have been cloned ${ }^{20-22}$. Rho GTPases participate in the regulation of a variety of cellular processes, e.g., gene expression, cell wall synthesis, $\mathrm{H}_{2} \mathrm{O}_{2}$ production, actin rearrangement processes, signal transduction pathways of MAP kinase ${ }^{23,24}$, and cytoskeletal assembly and reassembly, to produce a variety of cellular responses. As a regulatory factor, RhoGDI1 was significantly increased in A. fruticosa AM. Clearly, this protein is closely related to signal transduction between A. fruticosa and GM.

Multiple somatic embryogenesis receptor-like kinases (SERKs) have been defined, including the leucine-rich repeat receptor-like kinase (LRR-RLK) subfamily members and a family of transmembrane signal-transduction protein $s^{25}$. They are characterized by a predicted signal sequence, a single transmembrane region, and a cytoplasmic kinase domain. These features suggest that some SERK family protein kinases may play pivotal roles in communication between cells and the environment or in cell-cell interactions. Currently, SERK genes have been cloned from various plant species. The AtSERK3 gene participates in the brassinolide (brassinosteroid, BR) signal-transduction pathway. BR is an important hormone that regulates plant growth and development. Functional analysis has shown that the Arabidopsis thaliana mutant became a dwarf when the AtSERK3 gene is knocked out ${ }^{26}$. The overexpression of the OsSERK1 gene in rice cultivars leads to an increase in host resistance to blast fungus ${ }^{27}$; in contrast, transcripts of the lettuce LsSERK gene not only are decreased in in vitro somatic embryonic structures but also easily infect Sclerotinia ${ }^{28}$. Studies have also shown that the SERK gene is closely related to antibiotic stress. Plant root colonization by AMF results in increased levels of somatic embryogenesis receptor-like kinase, which plays a major role in promoting plant growth and enhancing plant disease resistance.

Stress and defense-related proteins. Inoculation with AMF has strong growth-promoting effects on A. fruticosa, especially at the mature stage of symbiont formation. These effects are mediated by increased action of SERK in BR signal-transduction pathways, which have a key role in the regulation of autoimmune responses and of plant root cell elongation and division. However, such regulation is not determined by a single factor. At an early stage of symbiosis, a weak defense response emerges when roots are stimulated by AMF colonization. Lectin plays a crucial role in this defense response by recognizing and binding to the sugar molecules of intruders and interfering with their function on plants. Many plant lectins can bind to glucose, mannitol, galactose or other monosaccharides, and they exhibit high affinity to the oligosaccharides of alien plants. Studies have shown that lectins on leguminous tree surfaces can gather rhizobia around the roots ${ }^{29}$. As AMF infect the roots of A. fruticosa, plant defense responses are initiated, resulting in agglutinin-2 accumulation. Agglutinin-2 is an important factor for the identification of AMF, similarly to rhizobia. 
When A. fruticosa is colonized by AMF, the abscisic acid (ABA) content increases rapidly, leading to the closing of plant stomata and decreased transpiration; this response also activates the genes encoding soluble osmolytes, thus decreasing stress injuries and the impact of stress-induced reactive oxygen and ethylene ${ }^{30}$. Therefore, ABA accumulation may stimulate metabolic enzymes to produce a feedback effect ${ }^{31}$. The major ABA catabolic route is decomposition via ABA $8^{\prime}$-hydroxylase to form phaseic acid. Therefore, ABA 8 '-hydroxylase accumulation in A. fruticosa may represent a mechanism for regulating ABA levels.

In multiple rice mapping populations, germin-like protein (GLP) markers have been associated with quantitative trait loci (QTL) for resistance to rice blast pathogens. At the early stage of rice blast fungus infection or mechanical damage, some OsGLPs are transiently induced and expressed. Varying $5^{\prime}$ regulatory regions and the differential expression of some protein family members between resistant and susceptible cultivars correspond with differential hydrogen peroxide $\left(\mathrm{H}_{2} \mathrm{O}_{2}\right)$ accumulation levels after fungal infection ${ }^{32}$. Wang discovered a new wheat germin-like protein ${ }^{33}$ that is up-regulated in both resistant and susceptible plants. It has been speculated to be involved in wheat defense responses. GLP is significantly increased at the early stage of AMF infection in roots of A. fruticosa, and it may participate in biotic stress responses.

Protein folding and degradation-related proteins. During the symbiosis process, the modification and degradation of peptides and proteins are critical for maintaining cell function. Protein disulfide isomerase, bi-ubiquitin, serine carboxypeptidase, proteasome subunit beta type- 6 and subtilisin-like protease SDD1 accumulate in plant roots to ensure proper cell function.

Plants use the proteasome pathway for selective protein degradation, and the proteasome plays pivotal roles in removing abnormally modified proteins and non-targeted proteins. Interactions between bi-ubiquitin and proteasome subunit beta type- 6 provide an effective way to degrade proteins. Bi-ubiquitin is highly conserved in eukaryotes, and it is covalently bound to target proteins through post-translational modification to mediate degradation.

Serine carboxypeptidase (SCP), an enzyme that catalyzes the hydrolysis of proteins in eukaryotes, has been found in rice, Arabidopsis and peas. It has been shown that SCP has broad functions in plants, including protein turnover and secondary metabolism synthesis, and it plays an important role in improving plant stress resistance. Liu showed that the expression of OsBISCPL1 was induced by rice blast fungi and antiviral signaling molecules (salicylic acid and jasmonic acid) ${ }^{34}$ and that overexpression of OSBISCPL1 could enhance disease resistance, oxidative stress tolerance and ABA sensitivity in transgenic Arabidopsis plants. OsBISCPL1 is expressed ubiquitously and differentially in rice, and it is induced by antiviral signaling molecules (BTH, JA, SA and ACC) and is up-regulated by incompatible interactions between rice and the blast fungus. Liu has shown that the expression of the ZmSCP gene in corn is up-regulated under induction by Rhizoctonia solani and that the ZmSCP protein are associated with various abiotic stresses ${ }^{35}$.

The subtilisin-like protease SDD1 is a member of the processing-type proteases in eukaryotes. As a preproprotein, it can direct peptides for transport to the cytoplasm. SDD1 is a crucial gene that regulates stomatal development and encodes a subtilisin-like serine protease. As a processive enzyme, it may activate a protein molecule or a signal that directs receptors into contact with epidermal cells during stomatal development processes. Liang has shown that the serine protease-encoding gene SDD1 is widely expressed acts on the development of stomata and is also necessary for normal root development ${ }^{36}$.

Protein disulfide isomerase (PDI), a multifunctional protein, is distributed widely in eukaryotic organisms and is involved in modifying/folding newly synthesized proteins. The catalytic thiol-disulfide exchange reaction to form disulfide is involved in many physiological processes, such as auxiliary protein folding in the endoplasmic reticulum, reconstruction of misfolded proteins, and the repair and refolding of damaged proteins under stress ${ }^{37}$. Additionally, as a chaperone, PDI can assemble heterogeneous protein peptides and regulate disulfide bonds in an ATP-dependent manner, and it may also be closely related to sugar transport, protein synthesis and other metabolic processes in eukaryotic organisms.

Energy-related Proteins. During the symbiosis process, dihydrolipoyl dehydrogenase, aldehyde dehydrogenase and isocitrate dehydrogenase [NAD] regulatory subunit 1 accumulated in plant roots. AMF colonization significantly enhances the energy metabolism of plants. The Krebs cycle provides more energy than glycolysis, and it is an important pathway not only an important for sugar metabolism but also for the metabolism of lipids, proteins and nucleic acids, which are eventually oxidized to carbon dioxide and water. Isocitrate dehydrogenase (IDH) is considered to be the rate-limiting enzyme of the Krebs cycle; it catalyzes decarboxylation to ketoglutarate while reducing $\mathrm{NAD}^{+}$to $\mathrm{NADH}^{38}$. Therefore, the activity of NAD-IDH has a significant impact on cellular metabolism. Isocitrate dehydrogenase [NAD] regulatory subunit 1, a regulatory factor, controls the activity of NAD-IDH and thus affects metabolic activity.

Kuhlemeier has explored the energy metabolism of tobacco pollen and has found that, in vegetative tissues ${ }^{39}$, pyruvate enters the Krebs cycle by pyruvate dehydrogenase (PDH); however, in reproductive organs, it is converted to acetaldehyde by pyruvate decarboxylase (PDC) and then enters the Krebs cycle via aldehyde dehydrogenase (ALDH) and acetyl coenzyme A synthetase (ACS). Thus, ALDH plays an important role in the pyruvate metabolism pathway of PDC/ALDH/ACS. Under stress conditions, plant cells quickly accumulate excessive reactive oxygen species (ROS), which cause oxidative stress and result in the accumulation of large amounts of toxic substance and eventually in plant death ${ }^{40}$. Aldehydes are an important component of peroxidation reaction products, and they play a crucial role in the oxidation of carboxylic aldehydes, the removal of toxic aldehydes, and the reduction of lipid peroxidation, thereby improving plant tolerance ${ }^{41}$. As an important member of the pyruvate dehydrogenase family, dihydrolipoyl dehydrogenases ensure the production of oxidatively decarboxylated pyruvate CoA, and CoA then enters the Krebs cycle to produce large amounts of energy for plant growth. 
Cellular structure-related proteins. Dramatic changes in plant morphology and in the penetrating mycelium, dynamic reorganization of cytoskeletal elements and organelle transformation occur when arbuscular vesicles develop ${ }^{42}$. Tubulin is an important component of the cytoskeleton, and it plays an important role in maintaining intracellular structural order and cell morphology. Meanwhile, tubulin is closely related to cellular transport, cell differentiation, cell motility, signal recognition, cell division and other developmental activities. Mills has revealed dramatic changes in both microtubules and actin arrangement in the host cell, and further studies have found that microtubules and actin rearrangement in the host cell are necessary for expression in non-host plants ${ }^{43}$. Studies on plant tubulin have primarily been focused on annual plants, such as Arabidopsis, tobacco, and rice, but study of the tubulin gene in perennial trees has been rare ${ }^{44}$.

Membrane and transport-related proteins. A $\mathrm{K}^{+}$efflux antiporter and coatomer, which are membrane and transport-related proteins, respectively, were found in the $A$. fruticosa mycorrhizea. The $\mathrm{K}^{+}$efflux antiporter is mainly responsible for maintaining the intracellular ion balance and regulating the cells' osmotic pressure. During AMF colonization, AMF invasion affects the ion balance of plant root cells, and plants maintain the intracellular ion balance to stimulate $\mathrm{K}^{+}$increases. Coatomer, a coat protein, transports vesicles, and vesicle-mediated non-selective transport ensures the accurate transport of proteins and lipids.

Metabolism-related proteins. During mycorrhizal symbiosis, increased levels of 3-oxoacyl -[acyl-carrier-protein] synthase, neutral ceramidase and caffeic acid 3-O-methyltransferase (COMT), which are metabolism-related proteins, were observed.

Lipid metabolism is one of the basic metabolic pathways in plants. The $\beta$-ketoacyl-acyl carrier protein synthase (KASI)-mediated acyl chain extension is important in the de novo synthesis of fatty acids. Ceramides, which are central molecules in the sphingolipid signaling pathway, play important roles as second messengers in plants and participate in many significant plant signaling pathways, such as cell growth, proliferation, differentiation, senescence and apoptosis ${ }^{45}$. Neuraminidase is a key enzyme that regulates ceramide. Neutral neuraminidase hydrolyzes ceramide to form sphingosine (ref). Liu has found that AtCER is involved in $\mathrm{H}_{2} \mathrm{O}_{2}$-induced oxidative stress ${ }^{46}$.

During cell morphogenesis, lignin plays an important role in the growth and development of vascular tissues and is involved in cell wall lignification, which increases the hardness or compressive strength of the cell wall. It also promotes the formation of mechanical tissues while also having a major impact on plant lodging, disease and stress resistance ${ }^{47}$. There are 3 types of monomeric lignin biosynthesis pathways,: the shikimate pathway, the phenylketonuria pathway and the lignin biosynthesis-specific pathway ${ }^{48}$. COMT is a key enzyme in the specific lignin pathway and is involved in the synthesis of S-lignin ${ }^{49}$. AMF colonization enhanced the synthesis of woody amorpha lignin, thus affecting the growth and development of plants.

Transcription and protein synthesis-related proteins. Ribosomal proteins are important components of the ribosome, and they have important roles in translation efficiency and ribosome stability. They also participate in important cellular processes, such as DNA repair, apoptosis and regulation of gene expression; e.g., 40S ribosomal proteins showed significant accumulation in plant roots after AMF invasion.

Nascent polypeptide-associated complex subunit alpha (NAC), which is located at the top of the newly synthesized polypeptide, can reversibly bind to eukaryotic ribosomes and guide the correct distribution and translocation of newly synthesized polypeptides in the cell. The observed increases in $40 \mathrm{~S}$ ribosomal protein and NAC levels, combined with folding- and degradation-associated proteins, ensure the fast and accurate synthesis and distribution of AM symbiosis-related proteins.

Transcriptional regulation is an important aspect of the regulation of gene expression. The results show significant accumulation of histone $\mathrm{H} 3$ and histone $\mathrm{H} 4$ in the host plant roots. Nucleosomes constitute the basic unit of chromatin in eukaryotes. Histones, which are structural proteins of chromosomes, play important roles in DNA folding and packing, protecting DNA from digestive enzymes, and gene regulation, tumor formation, and apoptosis. The N-terminal amino acids of histones participate in acetylation, methylation, phosphorylation, ubiquitination and other covalent modifications. Studies have shown that histones may change the structure of chromatin via post-translational modifications, thus modulating gene expression ${ }^{50}$.

Unknown proteins. During AMF symbiosis, the expression levels of proteins within A. fruticosa roots were changed; some proteins disappeared, and new symbiosis proteins arose. The functional analysis of symbiotic proteins in A. fruticosa, a non-model plant, is not difficult. Because these proteins were differentially expressed in the symbiotic system, they are targets for future studies.

AM-A. fruticosa molecular regulation model. By using bioinformatics analysis, we found that mycorrhizal proteins were involved in several biological processes and cellular activities (Fig. 3), and we verified that the symbiosis formed between AMF and A. fruticosa is a uniform and harmonious result of symbiotic interactions.

\section{Methods}

G. mosseae (GM) was harvested from sorghum, which was supplied by the Ecology Laboratory of Heilongjiang University, by co-culturing for longer than 40 days. Inocula contained a mixture of the rhizosphere that consisted of AM fungal spores, hyphae and mycorrhizal fragments. The inocula contained approximately 500 spores per $20 \mathrm{~g}$.

Seedling culture. Seeds of A. fruticosa were purchased from the Academy of Agricultural Sciences of Heilongjiang Province. A. fruticosa seeds were sterilized with $0.4 \% \mathrm{~K}_{2} \mathrm{MnO}_{4}$ for $20 \mathrm{~min}$, rinsed, and then covered with a layer of white gauze to keep them moist. Germination was conducted in an incubator at $30^{\circ} \mathrm{C}$ for $60 \mathrm{~h}$ after 


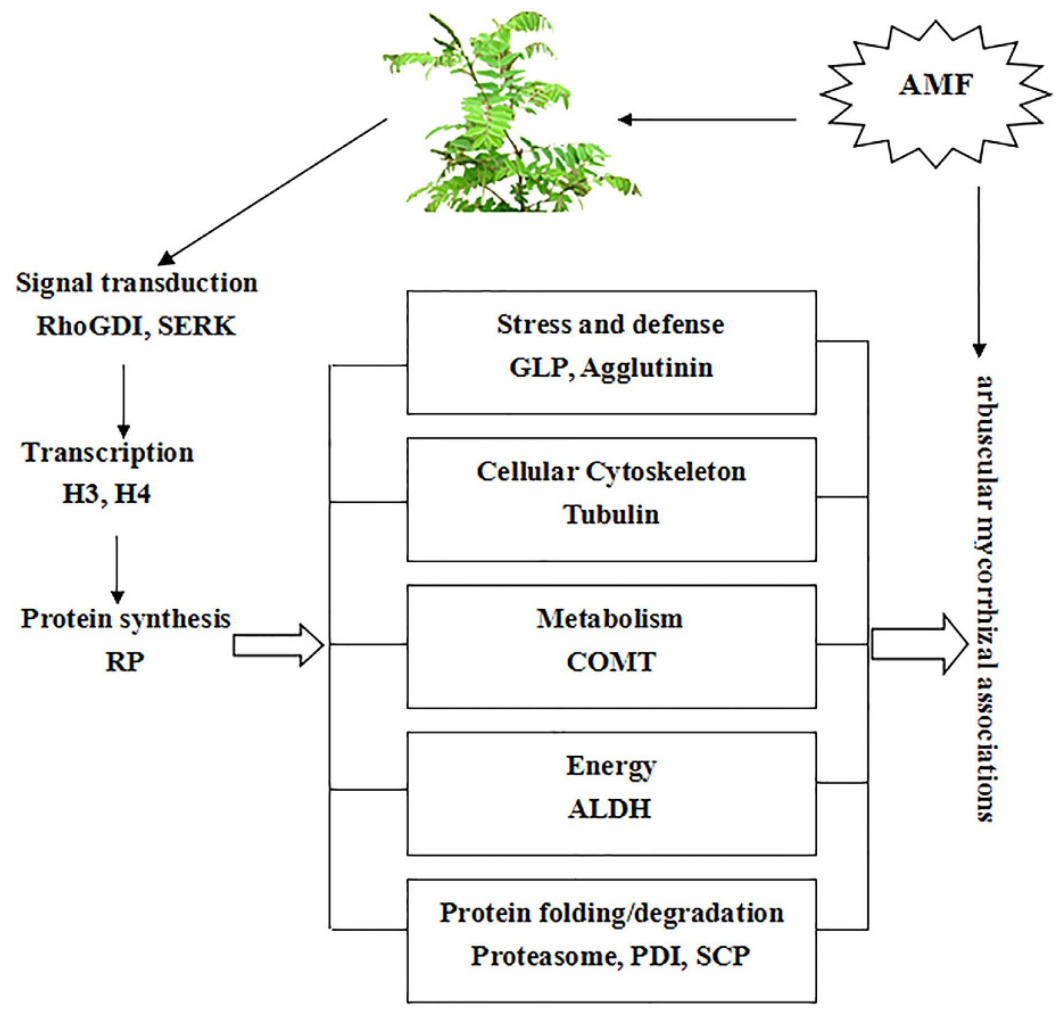

Figure 3. Several biological processes of cellular activities, and it was drawn by dandan Qi.

soaking for $24 \mathrm{~h}$. The growing medium was $50 \%$ peat soil, $30 \%$ vermiculite and $20 \%$ sand. It was sterilized in an autoclave at $121^{\circ} \mathrm{C}$ for $2 \mathrm{~h}$ and then air dried for 1 week before the start of the experiments ${ }^{10,11}$.

Mycorrhizal colonization percentage determination. The germinated seeds were then planted in a pre-sterilized mixed matrix and grown under a 16 -h photoperiod at temperatures of $25 / 18^{\circ} \mathrm{C}$ (day/night) with $60 \%$ relative humidity. One group was inoculated with GM inoculum, and the other was inoculated with sterilized inoculum as a control (CK). Each treatment was repeated 10 times. A total of 20 pots were arranged randomly and watered every 2 days. The mycorrhizal colonization percentage of the seedlings was determined using the Phillip and Hayman staining method (KOH bleaching-acid fuchsin stain) with some modifications (Phillips J M, 1970) ${ }^{51}$.

Protein extraction, protein quantification and SDS-PAGE. At the maturation stage, A. fruticosa roots were harvested, and total root protein was precipitated with $10 \%(\mathrm{w} / \mathrm{v})$ trichloroacetic acid (TCA) in acetone at $-20^{\circ} \mathrm{C}$ overnight. After centrifugation at $40,000 \times \mathrm{g}$ at $4{ }^{\circ} \mathrm{C}$ for $1 \mathrm{~h}$, the pellets were washed 3 times with cold $80 \%$ acetone. A 2-D Quant kit (GE Healthcare, USA) was used to determine the protein concentrations. SDS-polyacrylamide gel (12\%) electrophoresis was performed with $30-\mu \mathrm{g}$ samples at $120-\mathrm{V}$ constant voltage for $2 \mathrm{~h}$. The gel was stained with Coomassie blue and visualized ${ }^{52,53}$.

iTRAQ labeling. The CK group and the GM group each included 3 biological replicates. After digesting with trypsin, the proteins from the non-infected and infected samples were labeled with iTRAQ reagents 115 (CK1), 116 (CK2), 117 (CK3), 118 (GM1), 119 (GM2), and 121 (GM3) and were then combined following the manufacturer's protocol at a ratio of 1:1:1:1:1:1 for LC-MS/MS analysis ${ }^{54,55}$.

LC-MS/MS measurements. The labeled samples were pooled and purified using a strong cation-exchange chromatography (SCX) column and were then separated on an analytical column $(1.7 \mu \mathrm{m}, 100 \mu \mathrm{m} \times 100 \mathrm{~mm})$ at a flow rate of $300 \mathrm{~nL} / \mathrm{min}$ using a linear gradient of 5-35\% acetonitrile (ACN) over $40 \mathrm{~min}$. The ion spray voltage was $4.5 \mathrm{kV}$, and nitrogen was used as a nebulizing gas ( $30 \mathrm{psi})$ and a curtain gas (15 psi). From each MS scan, the 30 most intense precursor ions were selected for MS/MS fragmentation and were detected at a mass resolution of 30,000 at $\mathrm{m} / \mathrm{z} 400^{56}$. Data analysis was performed with a Triple TOF 5600 System, and then the iTRAQ data were compared with the protein sequences of homologous species after genome annotation.

Protein identification. Protein Pilot 4.0 (AB Sciex Inc., USA) was used to simultaneously identify and quantify proteins ${ }^{57,58}$. Differentially expressed proteins were required to satisfy 3 conditions for identification: (1) each confident protein identification involved at least 1 unique peptide; (2) the $P$-value was less than 0.05 ; and (3) changes of greater than 1.2 -fold or less than 0.8 fold were considered significant. All of the identified proteins were classified according to the annotations acquired by using the UniProt knowledge base and the GO database. 


\section{References}

1. Fitter, A., Heinemeyer, A. \& Staddon, P. The impact of elevated $\mathrm{CO}_{2}$ and global climate change on arbuscular mycorrhizas: a mycocentric approach. New Phytologist. 147, 179-187 (2000) .

2. Rosendahl, S. Populations and individuals of arbuscular mycorrhizal fungi. New Phytologist. 178, 253-266 (2008).

3. Smith, S. \& Read, D. Mycorrhizal symbiosis from cellular to ecosystem scales. Annual review of plant biology. 62, 227-250 (2008).

4. Smith, S. E. \& Smith, F. A. Roles of arbuscular mycorrhizas in plant nutrition and growth: new paradigms from cellular to ecosystem scales. Annual review of plant biology. 62, 227-250 (2011).

5. Kiers, E. T. et al. Reciprocal rewards stabilize cooperation in the mycorrhizal symbiosis. Science. 333, 880-882 (2011).

6. Trépanier, M. et al. Dependence of arbuscular-mycorrhizal fungi on their plant host for palmitic acid synthesis. Applied and environmental microbiology. 71, 5341-5347 (2005).

7. Recorbet, G. et al. Identification of in plant-expressed arbuscular mycorrhizal fungal proteins upon comparison of the root proteomes of Medicago truncatula colonised with two Glomus species. Fungal Genetics and Biology. 47, 608-618 (2010).

8. Aloui, A. et al. Arbuscular mycorrhizal symbiosis elicits shoot proteome changes that are modified during cadmium stress alleviation in Medicago truncatula. BMC plant biology. 11, 75 (2011).

9. Wang, Z. H., Yuan, K. \& Yang, L. F. Identification and Functional Analysis of Maize Leaf Proteins Responding to the Abuscular Mycorrhizal Fungi (AMF). Chinese Journal of Tropical Agriculture. 33, 40-44 (2013).

10. Song, F. Q., Li, J. Z. \& Zhang, X. X. Characterization of expressed genes in the establishment of arbuscular mycorrhiza between Amorpha fruticosa and Glomus mosseae. Journal of Forestry Research. 25, 541-548 (2014).

11. Song, F. Q., Kong, X. S., Li, J. Z. \& Chang, W. Screening the related genes in the AM Fungi and Amorpha fruticosa symbiosis with the subtractive hybridization technique. Scienta Slivae Sinicae. 50, 64-74 (2014).

12. Heikham, E., Rupam, K. \& Bhoopander, G. Arbuscular mycorrhizal fungi in alleviation of salt stress: a review. Annals of Botany. 104, $1263-1280$ (2009).

13. Song, F. Q., Kong, X. S., Dong, A. R. \& Liu, X. F. Impact of arbuscular mycorrhizal fungi on the growth and related physiological indexes of Amorpha fruticosa. Journal of Medicinal Plants Research. 6, 3648-3655 (2012).

14. Claudia, H. \& Helge, K. A roadmap of cell-type specific gene expression during sequential stages of the arbuscular mycorrhiza symbiosis. BMC Genomics. 14, 306-324 (2013).

15. Bonfante, P. et al. The Lotus japonicus LjSym4 gene is required for the successful symbiotic infection of root epidermal cells. Molecular plant-microbe interactions: MPMI. 13,1109-1120 (2000).

16. Novero, M. et al. Dual requirement of the LjSym4 gene for mycorrhizal development in epidermal and cortical cells of Lotus japonicus roots. New phytologist. 154, 741-749 (2002).

17. Bonfante, P. et al. At the interface between mycorrhizal fungi and plants: the structural organization of cell wall, plasma membrane and cytoskeleton. Fungal Associations. 9, 45-61 (2001).

18. Etienne Manneville, S. \& Hall, A. Rho GTPases in cell biology. Nature. 420, 629-635 (2002).

19. Li, H. et al. Arabidopsis Rho-related GTPases: differential gene expression in pollen and polar localization in fission yeast. Plant Physiology. 118, 407-417 (1998).

20. Winge, P., Brembu, T., Kristensen, R. \& Bones, A. M. Genetic structure and evolution of RAC-GTPases in Arabidopsis thaliana. Genetics. 156, 1959-1971 (2000).

21. Christensen, T. M. et al. Conserved subgroups and developmental regulation in the monocot rop gene family. Plant physiology. 133, 1791-1808 (2003).

22. Zheng, Z. L. \& Yang, Z. The Rop GTPase: an emerging signaling switch in plants. Plant molecular biology. 44, 1-9 (2000).

23. Takai, Y., Sasaki, T. \& Matozaki, T. Small GTP-binding proteins. Physiological reviews. 81, 153-208 (2001).

24. Yang, Z. et al. Small GTPases versatile signaling switches in plants. The Plant Cell. 14, 375-388 (2002).

25. Shiu, S. H. \& Bleecker, A. B. Receptor-like kinases from Arabidopsis form a monophyletic gene family related to animal receptor kinases. Proceedings of the National Academy of Sciences. 98, 10763-10768 (2001).

26. Noguchi, T. et al. Brassinosteroid-insensitive dwarf mutants of Arabidopsis accumulate brassinosteroids. Plant Physiology. 121, 743-752 (1999)

27. Hu, H., Xiong, L. \& Yang, Y. Rice SERK1 gene positively regulates somatic embryogenesis of cultured cell and host defense response against fungal infection. Planta. 222, 107-117 (2005).

28. Santos, M. et al. Suppression of SERK gene expression affects fungus tolerance and somatic embryogenesis in transgenic lettuce. Plant Biology. 11, 83-89 (2009).

29. Yin, A. H. \& Han, S. F. Relationship of Nodulation with Reactions of Letins of Leguminous Trees with EPS of Rhizobium. Journal of Nanjing Forestry University. 29, 88-90 (2005).

30. Verslues, P. \& Zhu, J. Plant signaling from genes to biochemistry. Biochemical Society Transactions. 33 (2005)

31. Cutler, A. J. \& Krochko, J. E. Formation and breakdown of ABA. Trends in plant science. 4, 472-478 (1999).

32. Davidson, R. M. et al. Rice germin-like proteins: Allelic diversity and relationships to early stress responses. Rice. 3, 43-55 (2010).

33. Wang, J. M. et al. Cloning and Chromosome Mapping of a Germin-Like Protein Gene in Wheat and Its Expression in Response to Infection with Wheat Powdery Mildew. Scientia Agricultura Sinica. 42, 3104-3111 (2009).

34. Liu, H. et al. A rice serine carboxypeptidase-like gene OsBISCPL1 is involved in regulation of defense responses against biotic and oxidative stress. Gene. 420, 57-65 (2008).

35. Liu, L. et al. Cloning and Expression Analysis of Serine Carboxypeptidases in Maize (Zea mays L.). Acta Agronomica Sinica. 39, 164-171 (2013).

36. Liang, K. et al. Identification and Genetic Analysis of Two Putative SDD1 Alleles in Arabidopsis. Chin Bull Bot. 3, 318-326 (2010).

37. Kim, Y. J. et al. Protein disulfide isomerase-like protein 1-1 controls endosperm development through regulation of the amount and composition of seed proteins in rice. PlOS one. 7, 44493 (2012).

38. Chen, R. D. \& Gadal, P. Structure, functions and regulation of NAD and NADP dependent isocitrate dehydrogenases in higher plants and in other organisms. Plant Physiology and Biochemistry. 28, 411-427 (1990).

39. Kuhlemeier, C. Aldehyde dehydrnase in tobacco pollen. Plant Mol Bio. 1, 355-365 (1997).

40. Mittler, R. Oxidative stress, antioxidants and stress tolerance. Trends in plant science. 7, 405-410 (2002).

41. Li, X. L., Yang, C. P. \& Xu, X. L. Molecular Cloning and Expression Analysis of Aldehyde Dehydrogenase (ALDH) Gene Segment in Leymus Chinensis. Chinese Agricultural Science Bulletin. 23, 115-120 (2007).

42. Genre, A. \& Bonfante, P. Actin versus tubulin configuration in arbuscule-containing cells from mycorrhizal tobacco roots. New phytologist. 140, 745-752 (1998).

43. Mills, D., Kunoh, H., Keen, N. \& Mayama, S. Molecular aspects of pathogenicity and resistance: requirement for signal transduction. American Phytopathological Society, 257-267 (1996).

44. Rao, G. D. \& Zhang, J. G. Advance of Studies on Plant Tubulin Gene. World Forestry Research. 26, 11-16 (2013).

45. Liang, H. et al. Ceramides modulate programmed cell death in plants. Genes \& Development. 17, 2636-2641 (2003).

46. Liu, R. H., Jiang, W. B. \& Yu, D. Q. The Response of AtCER to Oxidative Stress in Arabidopsis thaliana. Acta Botanica Yunnaica. 31, 326-334 (2009).

47. Li, B. et al. Advance on Key Enzyme Gene (COMT) Involved in Lignin Biosynthesis. Molecular Plant Breeding. 1, 117-124 (2010).

48. Hamada, K. et al. 4-Coumarate: coenzyme A ligase in black locust (Robinia pseudoacacia) catalyses the conversion of sinapate to sinapoyl-CoA. Journal of plant research. 117, 303-310 (2004). 
49. Kang, C. et al. Carbon Emission Flow in Networks. Sci. Rep. 2, 479, doi: 10.1038/srep00479 (2012).

50. O'Malley, D. M. et al. The role of laccase in lignification. The Plant Journa. 4, 751-757 (1993).

51. Wei, Q. D. et al. Advancement of Histone and Its Relationship with Regulation of Gene Expression. Journal of Tropical Medicine. 6, 596-598 (2006)

52. Phillips, J. \& Hayman, D. Improved procedures for clearing roots and staining parasitic and vesicular-arbuscular mycorrhizal fungi for rapid assessment of infection. Transactions of the British mycological Society. 55, 158-161 (1970).

53. Sophie, A., Leslie, M. H. \& Sona, P. ABA-Dependent and Independent G-Protein Signaling in Arabidopsis Roots Revealed through an iTRAQ Proteomics Approach. Journal of proteome research. 10, 3107-3122 (2011).

54. Zhang, K. R., Carolyn, M., Charles, H. H. \& Michael, A. D. The Medicago truncatula Small Protein Proteome and Peptidome. Journal of Proteome Research. 5, 3355-3367 (2006).

55. Liu, J. Y. et al. Comparative analysis of proteomic profile at different development stages of Volvariella volvacea by iTRAQ-coupled 2D LC-MSMS. Microbiology China. 39, 853-864 (2012).

56. Pan, X. Q. et al. iTRAQ Protein Profile Analysis of Tomato Green-ripe Mutant Reveals New Aspects Critical for Fruit Ripening. Journal of Proteome Research. 13, 1979-1993 (2014).

57. Alexey, C., Consuelo, M. V., Neus, V. \& Roman, A. Z. Functional Identification of Target by Expression Proteomics (FITExP) reveals protein targets and highlights mechanisms of action of small molecule drugs. Scientific Reports. 5, 11176, doi: 10.1038/srep11176 (2015).

58. Jose, A. M. et al. Quantitative proteomic analysis of host-pathogen interactions: a study of Acinetobacter baumannii responses to host airways. BMC Genomics. 16, 422-443 (2015).

\section{Acknowledgements}

We sincerely thank Prof. sixue Chen for greatly improved the manuscript. This study was supported by National Natural Science Foundation of China (31070576 and 31270535), Natural Science Foundation of Heilongjiang Province of China (No. ZD201206), Excellent Youth Foundation of Heilongjiang Province of China (No. JC201306) and High-level Talents Support Program of Heilongjiang University (Ecological Restoration Team).

\section{Author Contributions}

F.S. designed the research, D.Q. wrote the main manuscript text of chinese and interpreted the proteomics data, X.K. and X.L. wrote the main manuscript text of english and edited language, Y.G., Z.Z. and Q.W. extract proteins. All authors reviewed the manuscript.

\section{Additional Information}

Supplementary information accompanies this paper at http://www.nature.com/srep

Competing financial interests: The authors declare no competing financial interests.

How to cite this article: Song, F. et al. Proteomic analysis of symbiotic proteins of Glomus mosseae and Amorpha fruticosa. Sci. Rep. 5, 18031; doi: 10.1038/srep18031 (2015).

This work is licensed under a Creative Commons Attribution 4.0 International License. The images or other third party material in this article are included in the article's Creative Commons license, unless indicated otherwise in the credit line; if the material is not included under the Creative Commons license, users will need to obtain permission from the license holder to reproduce the material. To view a copy of this license, visit http://creativecommons.org/licenses/by/4.0/ 